\title{
INDUÇÃO DO AMADURECIMENTO DE FRUTOS CÍTRICOS EM PÓS- COLHEITA COM A APLICAÇÃO DE ETHEPHON ${ }^{1}$
}

\author{
MARCIO CHRISTIAN SERPA DOMINGUES², ELIZABETH ORIKA ONO, JOÃO DOMINGOS RODRIGUES
}

RESUMO - O presente experimento foi conduzido no Departamento de Botânica - IB/Unesp, Botucatu-SP, com o objetivo de estudar o efeito da aplicação pós-colheita de Ethephon no desverdecimento de laranjas-doces (Citrus sinensis (L.) Osbeck) cultivar 'Hamlin' e 'Baianinha', consideradas cultivares precoces. Os frutos foram imersos por diferentes tempos em solução aquosa de Ethephon mais $0,05 \%$ do adjuvante Extravon ( $25 \%$ de Alquilfenol - poliglicoléter). Os frutos foram colocados em caixas de madeira, sobre bancada, em temperatura ambiente, onde permaneceram por 72 horas. Os tratamentos utilizados foram os seguintes: T1- testemunha (água); T2 Ethephon - $1000 \mathrm{mg} . \mathrm{L}^{-1} / 5$ minutos de imersão; T3 - Ethephon - $2000 \mathrm{mg} \cdot \mathrm{L}^{-1} / 5$ minutos de imersão; T4 - Ethephon - $3000 \mathrm{mg} . \mathrm{L}^{-1} / 5$ minutos de imersão; T5 - Ethephon - 4000 mg.L.- 5 minutos de imersão; T6 - Ethephon - 1000 mg.L $\mathrm{L}^{-1} / 10$ minutos de imersão; T7 Ethephon - 2000 mg.L. $\mathrm{L}^{-1} / 10$ minutos de imersão; T8 - Ethephon - $3000 \mathrm{mg} \cdot \mathrm{L}^{-1} / 10$ minutos de imersão; T9 - Ethephon - $4000 \mathrm{mg} . \mathrm{L}^{-1} /$ 10 minutos de imersão. O delineamento experimental adotado foi o de blocos ao acaso, com 9 tratamentos e 4 repetições. Após 72 horas, o experimento foi avaliado através das seguintes variáveis: teor de clorofila da casca, teor de sólidos solúveis $\left({ }^{0}\right.$ Brix $)$ e textura dos frutos, além da análise visual com a utilização de fotografias. Com isso, verificou-se que houve influência do Ethephon no processo de mudança da cor da casca de frutos cítricos em todos os tratamentos, diferenciado-se estatisticamente da testemunha, porém, na concentração de $4000 \mathrm{mg} . \mathrm{L}^{-1}$, houve início de necrose em alguns pontos da casca dos frutos. Assim, pode-se concluir que a melhor concentração foi a de $1000 \mathrm{mg} \cdot \mathrm{L}^{-1}$ de Ethephon, para acelerar o processo de desverdecimento ou mudança da coloração da casca de frutos de laranjas precoces cultivares 'Hamlin' e 'Baianinha'.

Termos para indexação: Citrus sinensis, pós-colheita, Ethephon, amadurecimento, desverdecimento.

\section{THE EFFECT OF POSTHARVEST APPLICATION OF ETHEPHON ON CHANGE PEEL COLOR OF SWEET ORANGE}

\begin{abstract}
The present work was carried out on IB/Unesp, Botucatu-SP, with the objective to study the effect of postharvest application of Ethephon on change peel color of sweet orange cv. 'Hamlin' and 'Baianinha'. The fruits was soaked in water solution of Ethephon(2-chloroethyl fosfonic acid) plus $0,05 \%$ of Extravon ( $25 \%$ Alquil phenol poliglycoleter) The applied treatments were: T1control (water); T2 -Ethephon - 1000 mg.L-1 $/ 5$ minutes soaked; T3 - Ethephon - 2000 mg.L-1/ 5 minutes soaked; T4 - Ethephon - 3000 mg.L.- $\mathrm{L}^{-1} 5$ minutes soaked; T5 - Ethephon - $4000 \mathrm{mg} . \mathrm{L}^{-1} / 5$ minutes soaked; T6 - Ethephon - $1000 \mathrm{mg} . \mathrm{L}^{-1} / 10$ minutes soaked; T7 Ethephon - 2000 mg.L.-1 / 10 minutes soaked; T8 - Ethephon - 3000 mg.L.- / 10 minutes soaked; T9 - Ethephon - 4000 mg.L.-1 / 10 minutes soaked. The experiment was set up in a split plot design, utilizing 4 replications and 9 treatments. After 72 hours, the experiment was evaluated by folowing variables: amount of chlorophyll skin, total soluble solids, texture and visual analysis with pictures. The results showed that Ethephon reduced peel chlorophyll content on fruits in all treatments of Ethephon, but hight rates like $4000 \mathrm{mg} . \mathrm{L}^{-1}$ started points of necrosis on skin. Fruit quality was not adversely affected by all treatments. So it was concluded that the best rate was $1000 \mathrm{mg} \cdot \mathrm{L}^{-1}$ to induce the decrease of peel chlorophyll content of 'Hamlim' and 'Baianinha' sweet orange.
\end{abstract}

Index terms: Citrus sinensis, postharvest, Ethephon, peel color.

\section{INTRODUÇÃO}

Os frutos de laranja, cultivares 'Baianinha' e 'Hamlim', são consideradas cultivares precoces em relação às outras variedades comerciais e são colhidas com coloração mais verde que amarela ("de vez"), e por isso recebem melhor preço, pois são as primeiras que atingem o amadurecimento, que ocorre entre os meses de março e abril, e, dependendo das condições climáticas, esse processo pode ser acelerado ou retardado.

Em função desta precocidade e das altas temperaturas que ainda ocorrem nos messes do início do amadurecimento (março e abril), tais variedades não alcançam boa coloração de casca, sendo este um fator negativo para sua comercialização, pois frutos com coloração intensa (amarelo/laranja) alcançam preços melhores.

Para Chitarra \& Chitarra (1990), na fase de maturação fisiológica do fruto, este emerge de uma fase incompleta, atingindo crescimento pleno e máxima qualidade comestível, com o fruto ainda na planta. O amadurecimento, por sua vez, corresponde ao período final da maturação, durante o qual, o fruto apresenta-se completamente desenvolvido, com aparência externa e qualidade interna para consumo. O período subseqüente ao desenvolvimento do fruto é a senescência, durante a qual, o crescimento cessa, e os processos bioquímicos da senescência substituem as trocas químicas de amadurecimento, podendo ocorrer antes ou após a colheita dos

1 (Trabalho 208/2000). Recebido: 13/09/2000. Aceito para publicação: 21/09/2001.

2 Depto de Botânica - IB/UNESP, C.P. 510 - CEP: 18618-000 - Botucatu, SP. E-mail: sdomingues@laser.com.br 
frutos, ou mesmo durante a maturação.

Segundo Pantastico (1975) e Hulme (1970), os fenômenos relacionados com o amadurecimento incluem degradação das clorofilas a e b, degradação de pigmentos subjacentes e síntese de novos pigmentos, como os carotenóides, alterações de sabor, além da diminuição de acidez e adstringência, com elevação no teor de sólidos solúveis, mudanças na textura e conseqüente abscisão dos frutos, que são os outros fenômenos da maturação e senescência.

O Ethephon, quando aplicado em frutos cítricos, em pré ou pós-colheita, induz a formação do etileno, que acelera a degradação desta clorofila e a síntese de carotenóides, e, portanto, ocorre a mundança da coloração dos frutos da cor verde para alaranjada (Casas et al., 1989).

Nos frutos cítricos tratados com etileno, é observado que a atividade da clorofilase aumenta, promovendo a degradação da clorofila. Há redução no tamanho dos cloroplastos em frutos de tangerina cultivar 'Satsuma' tratados com etileno, que são diferentes do amadurecimento normal que ocorre com os frutos na planta. Estes resultados indicam que o caminho da degradação da clorofila em frutos tratados com etileno pode ser diferente dos caminhos da degradação da clorofila que ocorre no amadurecimento dos frutos na própria planta (Yamauchi et al., 1997).

Conforme relatam Chitarra \& Chitarra (1990), os frutos cítricos não possuem mecanismo bem definido de amadurecimento, onde o processo é gradual, e são denominados de não- climatéricos. Já os frutos de banana, maça e abacate, que são denominados de climatéricos, sofrem aumento repentino da taxa respiratória, efetivando, assim, o amadurecimento. Ao contrário, os frutos cítricos, por não serem climatéricos, amadurecem muito lentamente, mantendo níveis respiratórios constantes, que podem levar até vários meses.

Os frutos cítricos, quando removidos da planta, sofrem pequeno aumento na respiração e produção de etileno, assim, Abellles (1973) afirmou que o etileno pode ser utilizado no amadurecimento e desverdecimento ou somente na quebra da clorofila destes frutos, porém inúmeros fatores podem influenciar este processo..

Segundo Casas et al. (1989), além do momento adequado de aplicação dos reguladores vegetais, outros fatores podem influenciar os resultados a serem obtidos com a aplicação de Ethephon, como a variedade ou cultivar a ser utilizada, as condições climáticas no momento da aplicação e no período posterior. Existem ainda fatores que estão relacionados com a translocação de compostos nos frutos, que podem inibir ou diminuir os efeitos do Ethephon na mudança da coloração.

Oh et al. (1979), aplicando Ethephon em frutos colhidos de tangerina-'Satsuma', nas concentrações de 500 e 1000 mg.L.-1, obtiveram resultados de mudanças na coloração da casca dos frutos a partir de 5 dias após os tratamentos, ocorrendo desenvolvimento total da coloração 8 dias após, onde ocoreu a degradação completa da clorofila, sem prejudicar as qualidades interna e externa dos frutos, como sabor, textura e boa coloração da casca.

Além de tangerinas, o Ethephon pode ser aplicado também em laranjas-doces, pois Singh et al. (1978) aplicaram $1000 \mathrm{mg} . \mathrm{L}^{-1}$ de Ethephon, em laranja-'Hamlin', obtendo mudanças na coloração 8 a 10 dias após o tratamento, também sem prejuízos para a qualidade dos frutos, inclusive ocorrendo pequeno aumento na porcentagem de sólidos solúveis e diminuição da acidez dos frutos. Quando aplicada via gasosa, em câmaras fechadas apropriadas, a concentração de Ethephon pode ser reduzida pela metade, proporcionando o mesmo efeito de mudança na coloração da casca (Ahrens \& Barmore, 1987).

Com isso, o objetivo do presente trabalho foi acelerar o processo de amadurecimento, induzindo a mudança da coloração da casca, sem alteração na qualidade interna dos frutos, com aplicação pós-colheita de Ethephon, em laranjas-doces precoces cultivares 'Hamlin' e 'Baianinha'.

\section{MATERIAIS E MÉTODOS}

O presente experimento foi conduzido no Departamento de Botânica, Instituto de Biociências - Unesp/Botucatu, utilizando frutos colhidos precocemente de laranja (Citrus sinensis (L.) Osbeck) cultivares 'Baianinha' e 'Hamlin', com coloração ainda esverdeada ("de vez"), porém já fisiologicamente maduras, no município de Itapetininga-SP.

Os frutos foram submetidos aos tratamentos com Ethephon por imersão em solução durante diferentes tempos, e colocados em caixas de madeira à temperatura ambiente onde foram submetidos à análise qualitativa e coloração de fruto, após 72 horas.

Os tratamentos foram os seguintes:

T1 - Testemunha (água); T2 - Ethephon - 1000 mg.L $\mathrm{L}^{-1} / 5$ minutos de imersão;T3 - Ethephon - $2000 \mathrm{mg} \cdot \mathrm{L}^{-1} / 5$ minutos de imersão; T4 -Ethephon - 3000 mg.. $\mathrm{L}^{-1} / 5$ minutos de imersão; T5 - Ethephon - $4000 \mathrm{mg} . \mathrm{L}^{-1} / 5$ minutos de imersão; T6 - Ethephon - 1000 mg.L.-1 / 10 minutos de imersão; T7 - Ethephon - 2000 mg. $\mathrm{L}^{-1} / 10$ minutos de imersão; T8 - Ethephon - $3000 \mathrm{mg} . \mathrm{L}^{-1} / 10$ minutos de imersão; T9 - Ethephon - 4000 mg.L $\mathrm{L}^{-1} / 10$ minutos de imersão.

O produto comercial utilizado foi Ethrell, contendo 240 g.L $\mathrm{L}^{-1}$ de ácido 2- cloroetilfosfônico. Às soluções de tratamento, foi adicionado $0,05 \%$ de espalhante adesivo Extravon, contendo $25 \%$ de alquilfenol-poliglicoléter, diluídos em água.

Após 72 horas dos tratamentos, os frutos foram submetidos a avaliações qualitativas do teor de sólidos solúveis do suco, textura e coloração dos frutos com a determinação do teor de clorofila da casca.

O teor de clorofila foi determinado através do aparelho Minolta Chlorophyll meter, e os dados expressos em SPD (silicon photo diode). A determinação da textura foi realizada com o penetrômetro manual, sendo os dados expressos em g. $\mathrm{Kg}^{-1}$, e o teor de sólidos solúveis através de refratômetro ( $\left.{ }^{\circ} \mathrm{Brix}\right)$.

O delineamento experimental utilizado foi o de blocos ao acaso, com 9 tratamentos e 4 repetições, contendo 10 frutos cada uma.

\section{RESULTADOS E DISCUSSÃO}

Através da Tabela 1, pode-se verificar que houve efeito significativo dos tratamentos sobre o teor de clorofila da casca dos frutos de laranja cultivares 'Hamlin' e 'Baianinha'. A aplicação de Ethephon pós-colheita proporcionou alteração 
na coloração da casca dos frutos, ou seja, houve degradação da clorofila. Segundo Korban (1998), o etileno na forma de Ethephon é utilizado para estimular a degradação de clorofila e ativar a síntese de carotenóides em frutos de laranja e tangerina e, assim, utilizado comunmente no desverdecimento de frutos de citros. Pode-se observar, ainda, que tanto a imersão dos frutos no tratamento com Ethephon, nas diferentes concentrações por 5 ou 10 minutos, apresentaram resultados semelhantes no teor de clorofila da casca dos furtos.

Estes resultados concordam com Casas \& Llacér (1989), que relatam haver mudança na coloração devido à síntese de carotenóides em frutos de laranja, mostrando coloração laranja intensa após 72 horas do tratamento. Também neste trabalho, a coloração da casca dos frutos de laranja das duas cultivares estudadas eram intensas, 72 horas após os tratamentos com Ethephon em todas as concentrações empregadas.

De acordo com a Tabela 2, pode-se observar que não houve efeito significativo dos tratamentos sobre a textura dos frutos de laranja tanto na cultivar 'Hamlin' como na 'Baianinha' . Portanto, os frutos de laranja tratados com Ethephon não sofreram alteração na textura, não ocorrendo perda da qualidade dos frutos. Assim, frutos de laranja-'Hamlin' e 'Baianinha' continuaram firmes, após 72 horas da imersão nos tratamentos com Ethephon.

Foi observado também que, nas concentrações mais elevadas (2000 a $4000 \mathrm{mg} . \mathrm{L}^{-1}$ de Ethephon), houve início de necrose na casca dos frutos, principalmente na concentração de 4000 mg. $\mathrm{L}^{-1}$, podendo-se considerar esta concentração fitotóxica para os frutos.

Em relação ao teor de sólidos solúveis, pode-se verificar que houve efeito significativo dos tratamentos para a cultivar 'Hamlin', mas não para a cultivar 'Baianinha' (Tabela 3). Para frutos da cultivar 'Hamlin', o tratamento com Ethephon a 3000 mg. $\mathrm{L}^{-1}$ por 10 minutos foi significativamente maior que os

TABELA 1 - Teor de clorofila da casca (SPD) de frutos de laranja cultivares 'Hamlin' e 'Baianinha', imersos em solução de Ethephon, 72 horas após o tratamento.

\begin{tabular}{lcc}
\hline Tratamentos & 'Hamlin' & 'Baianinha' \\
\hline Testemunha & \multicolumn{2}{c}{ Teor de clorofila } \\
\hline Ethephon $-1000 \mathrm{mg} \cdot \mathrm{L}^{-1} / 5$ minutos & $0,28 \mathrm{a}$ & $5,24 \mathrm{a}$ \\
\hline Ethephon $-2000 \mathrm{mg} \cdot \mathrm{L}^{-1} / 5$ minutos & $0,71 \mathrm{~b}$ & $0,15 \mathrm{~b}$ \\
\hline Ethephon $-3000 \mathrm{mg} \cdot \mathrm{L}^{-1} / 5$ minutos & $0,00 \mathrm{~b}$ & $0,10 \mathrm{~b}$ \\
\hline Ethephon $-4000 \mathrm{mg} \cdot \mathrm{L}^{-1} / 5$ minutos & $0,00 \mathrm{~b}$ & $0,26 \mathrm{~b}$ \\
\hline Ethephon $-1000 \mathrm{mg} \cdot \mathrm{L}^{-1} / 10$ minutos & $0,00 \mathrm{~b}$ & $0,32 \mathrm{~b}$ \\
\hline Ethephon $-2000 \mathrm{mg} \cdot \mathrm{L}^{-1} / 10$ minutos & $0,00 \mathrm{~b}$ & $0,20 \mathrm{~b}$ \\
\hline Ethephon $-3000 \mathrm{mg} \cdot \mathrm{L}^{-1} / 10$ minutos & $0,98 \mathrm{~b}$ & $0,25 \mathrm{~b}$ \\
\hline Ethephon $-4000 \mathrm{mg} \cdot \mathrm{L}^{-1} / 10$ minutos & $0,35 \mathrm{~b}$ & $0,56 \mathrm{~b}$ \\
\hline DMS & 0,87 & 0,76 \\
\hline $\mathrm{CV}(\%)$ & 58,73 & 50,01 \\
\hline
\end{tabular}

Médias seguidas de mesma letra, na vertical, não diferem entre si, pelo teste Tukey, ao nível de $5 \%$ de probabilidade.
TABELA 2 - Textura da casca de frutos de laranja cultivar 'Hamlin' e 'Baianinha', imersos em solução de Ethephon, 72 horas após o tratamento.

\begin{tabular}{lcc}
\hline Tratamentos & 'Hamlin' & 'Baianinha' \\
\hline & \multicolumn{2}{c}{ Textura $\left(\mathrm{g} . \mathrm{Kg}^{-1}\right)$} \\
\hline Testemunha & $108.88 \mathrm{a}$ & $93.12 \mathrm{a}$ \\
\hline Ethephon $-1000 \mathrm{mg} \cdot \mathrm{L}^{-1} / 5$ minutos & $112.00 \mathrm{a}$ & $88.38 \mathrm{a}$ \\
\hline Ethephon $-2000 \mathrm{mg} \cdot \mathrm{L}^{-1} / 5$ minutos & $122.25 \mathrm{a}$ & $93.88 \mathrm{a}$ \\
\hline Ethephon $-3000 \mathrm{mg} \cdot \mathrm{L}^{-1} / 5$ minutos & $107.75 \mathrm{a}$ & $104.50 \mathrm{a}$ \\
\hline Ethephon $-4000 \mathrm{mg} \cdot \mathrm{L}^{-1} / 5$ minutos & $101.38 \mathrm{a}$ & $80.88 \mathrm{a}$ \\
\hline Ethephon $-1000 \mathrm{mg} \cdot \mathrm{L}^{-1} / 10$ minutos & $108.88 \mathrm{a}$ & $94.25 \mathrm{a}$ \\
\hline Ethephon $-2000 \mathrm{mg} \cdot \mathrm{L}^{-1} / 10$ minutos & $95.86 \mathrm{a}$ & $108.00 \mathrm{a}$ \\
\hline Ethephon $-3000 \mathrm{mg} \cdot \mathrm{L}^{-1} / 10$ minutos & $108.13 \mathrm{a}$ & $93.38 \mathrm{a}$ \\
\hline Ethephon $-4000 \mathrm{mg} \cdot \mathrm{L}^{-1} / 10$ minutos & $98.00 \mathrm{a}$ & $83.13 \mathrm{a}$ \\
\hline DMS & 49.15 & 28.73 \\
\hline CV $(\%)$ & 19.24 & 12.81
\end{tabular}

Médias seguidas de mesma letra, na vertical, não diferem entre si, pelo teste Tukey, ao nível de $5 \%$ de probabilidade.

TABELA 3 - Teor de sólidos solúveis ( ${ }^{\circ}$ Brix) de frutos de laranja cultivar 'Hamlin' e 'Baianinha', imersos em solução de Ethephon, 72 horas após o tratamento.

\begin{tabular}{lcc}
\hline Tratamentos & 'Hamlin' & 'Baianinha' \\
\hline & Teor de sólidos solúveis ('Brix) \\
\hline Testemunha & $9.40 \mathrm{abc}$ & $7.73 \mathrm{a}$ \\
\hline Ethephon - $1000 \mathrm{mg} \cdot \mathrm{L}^{-1} / 5$ minutos & $8.80 \mathrm{c}$ & $7.50 \mathrm{a}$ \\
\hline Ethephon - $2000 \mathrm{mg} \cdot \mathrm{L}^{-1} / 5$ minutos & $8.93 \mathrm{bc}$ & $8.27 \mathrm{a}$ \\
\hline Ethephon - $3000 \mathrm{mg} \cdot \mathrm{L}^{-1} / 5$ minutos & $8.93 \mathrm{bc}$ & $7.93 \mathrm{a}$ \\
\hline Ethephon - $4000 \mathrm{mg} \cdot \mathrm{L}^{-1} / 5$ minutos & $9.40 \mathrm{abc}$ & $7.20 \mathrm{a}$ \\
\hline Ethephon - $1000 \mathrm{mg} \cdot \mathrm{L}^{-1} / 10$ minutos & $8.87 \mathrm{bc}$ & $7.93 \mathrm{a}$ \\
\hline Ethephon - $2000 \mathrm{mg} \cdot \mathrm{L}^{-1} / 10$ minutos & $9.93 \mathrm{abc}$ & $7.93 \mathrm{a}$ \\
\hline Ethephon - 3000 mg.L $\mathrm{L}^{-1} / 10$ minutos & $10.33 \mathrm{a}$ & $7.73 \mathrm{a}$ \\
\hline Ethephon - $4000 \mathrm{mg} \cdot \mathrm{L}^{-1} / 10$ minutos & $10.06 \mathrm{ab}$ & $7.33 \mathrm{a}$ \\
\hline DMS & 1.26 & 1.20 \\
\hline CV $(\%)$ & 4.63 & 5.33
\end{tabular}

Médias seguidas de mesma letra, na vertical, não diferem entre si, pelo teste Tukey, ao nível de $5 \%$ de probabilidade.

tratamentos com Ethephon a 1000, 2000 e $3000 \mathrm{mg} \cdot \mathrm{L}^{-1}$ por 5 minutos e com Ethephon a $1000 \mathrm{mg} . \mathrm{L}^{-1}$ por 10 minutos, ou seja, o teor de açúcares foi menor nas menores concentrações de Ethephon utilizadas por curto tempo de imersão. Em geral, para esta cultivar, o tratamento dos frutos com altas concentrações de Ethephon, 3000 e $4000 \mathrm{mg} . \mathrm{L}^{-1}$, durante 10 minutos, embora sem diferir da testemunha, tendeu a apresentar maiores teores de açúcares, fato também observado por Singh et al. (1978), discordando de Fisher \& Monselise (1971), que também verificaram que o Ethephon acelera a degradação da clorofila, mas não afeta a qualidade interna dos frutos.

Já, frutos da cultivar 'Baianinha' não apresentaram teor de sólidos solúveis totais alterado pelos tratamentos com Ethephon nas diferentes concentrações e tempo de tratamento; portanto, foram menos sensíveis ao tratamento com Ethephon.

Para antecipar a obtenção de frutos de laranjeira-'Hamlin' 
e 'Baianinha' com coloração da casca alaranjada, o tratamento com Ethephon torna-se uma técnica muito útil. Pelos resultados obtidos no presente trabalho, pode-se verificar que a concentração de 1000 mg. $\mathrm{L}^{-1}$ de Ethephon, por um tempo de imersão de 5 minutos, já é eficiente para a degradação total da clorofila sem alteração da textura da casca e do teor de sólidos solúveis. As demais concentrações e o tempo de imersão de 10 minutos também foram efetivos na degradação da clorofila, confirmando as informações de Casas \& Llacér (1989), que o etileno formado acelera a degradação da clorofila em frutos cítricos sem afetar a qualidade interna dos frutos.

As concentrações de 2000 a 4000 mg. $\mathrm{L}^{-1}$ de Ethephon apresentaram resposta semelhante à concentração de $1000 \mathrm{mg.L}$ ${ }^{1}$, não se justificando a utilização destas altas concentrações que, além de aumentar o custo do tratamento, podem causar lesão na casca dos frutos, inviabilizando a sua comercialização.

\section{CONCLUSÃO}

De acordo com os resultados obtidos durante o presente experimento, podemos concluir que a aplicação de Ethephon, na concentração de $1000 \mathrm{mg} . \mathrm{L}^{-1}$, em pós-colheita de laranjas-doces precoces, cultivares 'Hamlin' e 'Baianinha', foi a mais viável para acelerar a mudança da coloração da casca, sem alterar a qualidade interna dos frutos.

\section{REFERÊNCIAS BIBLIOGRÁFICAS}

ABELES, F.B. Ethylene in plant biology. New York: Academic Press, 1973. 302p.

AHRENS, M.J.; BARMORE, C.R. Interactive effects of temperature and ethylene concentration on postharvest color development in citrus. Acta horticulture, Wagenengin, n.201, p.21-27, 1987.

CASAS, A.; LLACÉR, J. El color de los frutos cítricos. III. Modificatión del color mediante tratamientos pre-recolección.
Revista de Agroquímica y Tecnología de almentos, Valencia, v.29, n.2,p. 173-190, 1989.

CHITARRA, M.I.F.; CHITARRA, A.B. Pós-colheita de frutos e hortaliças: fisiologia e manuseio. Lavras: ESAL/FAEPE, 1990. $293 p$.

FISHER, M.; MONSELISE, S.P. The use of ethephon (2chloroethylphosphonic acid) to promote color development of Shamouti orange fruit. Israel Journal of Agriculture, v.21, p.67, 1971.

HULME, A.C. (Ed.), The biochemistry of fruits and their products. London: Academic Press, 1971.

KORBAN, S.S. Influence of growth regulators on fruit plant growth and development. In: CONGRESSO BRASILEIRO DE FRUTICULTURA, 15., 1998, Poços de Caldas. Conferências... Poços de Cladas: Sociedade Brasileira de Fruticultura, 1998. p. 56-81.

OH, S.D.; KIM, Y.Y.; HONG, S.B.; CHUNG, S.K. Effect of postharvest application of ethephon, ethylene and methionine on colour and quality of satsuma. Journal of the Korean Society for Horticultural Science, Suwon, v.20, n.2, p.142-147, 1979.

PANTASTICO, E. R. B. Postharvest physiology handling and utilization of tropical and subtropical fruits and vegetables. West Port: Avi, 1975. 560p.

SINGH, H. P.; SRIVASTAVA, K.C.; GANAPATHY, K.M.; MUTHAPPA, D.P.; RANDHAWA, G.S. Effect of ethephon (2chloro ethyl phosphonic acid) on postharvest degreening of mandarin and sweet orange. Vatika, v.1, n.1, p.56-63, 1978.

YAMAUCHI, N.; AKIYAMA, Y.; KAKO, S.; HASHINAGA, F. Chlorophyll degradation in Wase satsuma mandarin (Citrus unshiu Marc.) fruit with on-tree maturation and ethylene treatment. Scientia Horticulture, Wagenengin, v.71, p. 35-42, 1997. 